\title{
Short-course neoadjuvant in situ vaccination for murine melanoma
}

\author{
Taylor J Aiken, ${ }^{1}$ David Komjathy, ${ }^{2}$ Matthew Rodriguez, ${ }^{2}$ Ashley Stuckwisch, ${ }^{2}$ \\ Arika Feils, ${ }^{2}$ Vladimir Subbotin, ${ }^{2,3}$ Jen Birstler, ${ }^{4}$ Stephen D Gillies, ${ }^{5}$ \\ Alexander L Rakhmilevich, ${ }^{2}$ Amy K Erbe, ${ }^{2}$ Paul M Sondel (D) ${ }^{2,6}$
}

To cite: Aiken TJ, Komjathy D, Rodriguez M, et al. Short-course neoadjuvant in situ vaccination for murine melanoma. Journal for ImmunoTherapy of Cancer 2022;10:e003586. doi:10.1136/ jitc-2021-003586

- Additional supplemental material is published online only. To view, please visit the journal online (http://dx.doi.org/10. 1136/jitc-2021-003586).

Accepted 11 December 2021

D Check for updates

(C) Author(s) (or their employer(s)) 2022. Re-use permitted under CC BY-NC. No commercial re-use. See rights and permissions. Published by BMJ.

${ }^{1}$ Department of Surgery, University of Wisconsin, Madison, Wisconsin, USA ${ }^{2}$ Department of Human Oncology, University of Wisconsin, Madison, Wisconsin, USA

${ }^{3}$ Arrowhead Pharmaceuticals Inc, Madison, Wisconsin, USA ${ }^{4}$ Biostatistics and Medical Informatics, University of Wisconsin-Madison, Madison, Wisconsin, USA

${ }^{5}$ Provenance

Biopharmaceuticals, Carlisle,

Massachusetts, USA

${ }^{6}$ Department of Pediatrics, University of Wisconsin, Madison, Wisconsin, USA

Correspondence to

Dr Paul M Sondel;

pmsondel@humonc.wisc.edu

\section{ABSTRACT}

Background Surgical resection remains an important component of multimodality treatment for most solid tumors. Neoadjuvant immunotherapy has several potential advantages, including in-situ tumor vaccination and pathologic assessment of response in the surgical specimen. We previously described an in-situ tumor vaccination strategy in melanoma using local radiation (RT) and an intratumoral injection of tumor-specific antiGD2 immunocytokine (IT-IC). Here we tested whether neoadjuvant in-situ tumor vaccination using anti-GD2 immunocytokine and surgical resection, without RT, could generate immunologic memory capable of preventing recurrence or distant metastasis.

Methods Mice bearing GD2 expressing B78 melanoma tumors were treated with neoadjuvant radiation, IT-IC, or combined RT + IT-IC. Surgical resection was performed following neoadjuvant immunotherapy. Immune infiltrate was assessed in the resection specimens. Mice were rechallenged with either B78 contralateral flank tumors or pulmonary seeding of non-GD2 expressing B16 melanoma metastasis induced experimentally. Rejection of rechallenge in mice treated with the various treatment regimens was considered evidence of immunologic memory.

Results Neoadjuvant IT-IC and surgical resection resulted in increased CD8 T cell infiltration, a higher CD8:regulatory $T$ cell ratio, and immunologic memory against contralateral flank rechallenge. The timing of resection did not significantly impact the development of memory, which was present as early as the day of surgery. There was less local wound toxicity with neoadjuvant IT-IC compared with neoadjuvant RT + IT IC. Neoadjuvant IT-IC and resection resulted in the rejection of $\mathrm{B} 16$ lung metastasis in a $\mathrm{CD} 4 \mathrm{~T}$ cell dependent manner.

Conclusions Neoadjuvant IT-IC generates immunologic memory capable of preventing distant metastasis despite limited efficacy against large primary melanoma tumors. By combining neoadjuvant tumor vaccination and surgery, the toxicity of local RT was avoided. These preclinical data support further investigation regarding the use of neoadjuvant IT-IC in patients with melanoma at high risk for occult distant disease.

\section{INTRODUCTION}

Surgical resection remains a key component of multimodality treatment for most solid tumors, however local and distant recurrence are major sources of mortality following successful resection. Neoadjuvant chemotherapy and radiation have been shown to reduce recurrence in multiple cancer types. ${ }^{1-4}$ Potential benefits of neoadjuvant therapy include debulking of the primary tumor to facilitate complete resection, earlier systemic therapy for occult distant disease, and pathologic assessment of therapeutic response. ${ }^{35}$ Immunotherapy may have unique benefits in the preoperative setting, where the in-situ tumor can provide an abundant source of tumor antigen for endogenous T cell priming in vivo. ${ }^{6}$

Neoadjuvant immunotherapy has been well described at the preclinical level. Liu et aldemonstrated that neoadjuvant checkpoint inhibition is superior to adjuvant checkpoint inhibition in $4 \mathrm{~T} 1.2$ breast cancer, leading to improved survival and a more robust $\mathrm{T}$ cell response. ${ }^{7}$ The authors also demonstrated that short-course immunotherapy immediately before surgical resection was superior to a longer duration between therapy initiation and surgery. ${ }^{8}$ Beyond checkpoint inhibition, tumor vaccination strategies have been studied in the neoadjuvant setting with multiple studies demonstrating superior results of neoadjuvant compared with adjuvant tumor vaccination. ${ }^{910}$

We have previously described an in-situ tumor vaccination strategy targeting GD2, a disialoganglioside found to be expressed on neuroblastoma and melanoma. ${ }^{11}{ }^{12}$ This strategy utilizes a fusion protein consisting of the hu14.18 anti-GD2 monoclonal antibody linked to interleukin-2 (IL2). Termed an immunocytokine (IC), this fusion protein can be delivered intratumorally (IT), generating a potent T cell response. ${ }^{11} 13$ This has been demonstrated previously with IT IL-2 and other nonGD2 based ICs. ${ }^{14-16}$ Furthermore, we showed that when radiation (RT) was combined with the hu14.18-IL2 intratumoral immunocytokine (IT-IC), it was effective in curing mice 
bearing GD2-expressing B78 melanoma tumors. ${ }^{11}$ The specificity and some of the mechanisms of the antitumor response by B78 melanoma to IT-IC and to RT + IT-IC have been reported previously. ${ }^{13}$ These reports demonstrated the following: (1) IT-IC is more potent than intravenous IC, and more potent than IT-IL2; (2) RT + IT IC is more potent than RT + IT-anti-GD2 mAb; (3) the ability of RT + IT-IC to cure mice of B78 depends on T cells; and (4) the ability of mice cured of B78 following RT + IT-IC treatment to specifically reject a rechallenge of B78 melanoma is also T cell dependent. However, large B78 tumors are not effectively treated by IT-IC monotherapy due to progression of the primary tumor. ${ }^{11}$

We hypothesized that IT-IC might be effective when given as neoadjuvant immunotherapy. Though less effective against bulky primary tumors, neoadjuvant IT-IC might provide sufficient efficacy against distant disease though effective $\mathrm{T}$ cell priming while avoiding the potential toxicity of combined RT and IT-IC. In addition, surgical reduction of primary tumor burden has been shown to improve immunologic response and survival from metastatic disease in mouse models, potentially increasing the efficacy of IT-IC mediated tumor vaccination. ${ }^{17}$ In this report we test whether neoadjuvant IT-IC and surgical resection induces an effective antitumor immune response capable of preventing distant recurrence.

\section{MATERIALS AND METHODS \\ Cells}

B78-D14 (B78) melanoma is a poorly immunogenic cell line derived from B78-H1 cells, which were originally derived from B16 melanoma. ${ }^{18-20} \mathrm{~B} 78-\mathrm{D} 14$ cells have functional GD2/GD3 synthase and express the disialoganglioside GD2. ${ }^{18}{ }^{19}$ The B16 cell line lacks GD2 expression. ${ }^{19}$ B78 and B16 cells were grown in RPMI 1640 supplemented with $10 \%$ heat-inactivated fetal bovine serum, 2 $\mathrm{mmol} / \mathrm{L}$ l-glutamine, $100 \mathrm{U} / \mathrm{mL}$ penicillin, and $100 \mathrm{\mu g} /$ $\mathrm{mL}$ streptomycin. Both cell lines were confirmed to be negative for mycoplasma by PCR prior to use. Cell authentication was performed per the American Type Culture Collection guidelines prior to use. Cells were cultured for less than five passages prior to use. The B16 cell line was transduced via lentivirus to express luciferase using a pLVTurboRFP/Luciferase/puromycin plasmid (VB1807251102ser, VectorBuilder), and stable cell lines were cloned to generate 'B16-luc'. B16-luc was used to monitor tumor growth in our pulmonary metastasis model. B16 cells were transduced to express GD2 via lentiviral transduction with GD2 and GD3 synthase under puromycin (4 $\mathrm{ug} / \mathrm{mL})$ and blasticidin $(7.5 \mathrm{ug} / \mathrm{mL})$ selection, respectively (VB170105-1067pah, VB170104-1118qqc; VectorBuilder), and stable cell lines were cloned to generate 'B16-GD2'.

\section{Murine tumor models}

All mice procedures were conducted in accordance with the Institutional Animal Care and Use Committee at the
University of Wisconsin-Madison. C57BL/6 female mice aged 6-8 weeks were purchased from Taconic. B78 flank tumors were engrafted by intradermal flank injection of $2 \times 10^{6}$ tumor cells diluted in $100 \mu \mathrm{L}$ phosphate buffed saline (PBS). Tumor size was determined by precision caliper measurement and tumor volume was approximated using the formula (tumor volume in $\mathrm{mm}^{3}=\left((\text { tumor length in } \mathrm{mm})^{2} \times(\right.$ tumor width in $\mathrm{mm}) / 2$. Mice were randomized to treatment groups when tumors reached enrollment size $\left(50-100 \mathrm{~mm}^{3}\right)$, which normally required 2-4 weeks following initial tumor implantation. The first day of treatment with IT-IC was defined as 'day 1'. Approximately $10 \%$ of mice developed tumors that were not in the size range for enrollment eligibility at the time of randomization after initial implantation (ie, too small or too large to be within the tumor volume range of (50-100 $\left.\mathrm{mm}^{3}\right)$ ) and these mice were excluded from randomization.

Flank tumor rechallenge experiments were performed in tumor-free mice approximately 40 days after treatment start unless otherwise indicated. Rechallenge consisted of subcutaneous flank injection of $2 \times 10^{6} \mathrm{~B} 78$ tumor cells on the contralateral flank from the original tumor cell injection. Tumor volume was assessed two times per week by a blinded observer, and mice were euthanized when tumors exceeded $20 \mathrm{~mm}$ in any direction or mice were assessed to be in distress by changes to posture, activity, or grooming.

B16-luc pulmonary metastases were obtained by tail vein injection of $2 \times 10^{5}$ tumors cells diluted in $100 \mu \mathrm{L}$ PBS. Animals were monitored weekly by bioluminescent imaging with Perkin Elmer IVIS Spectrum In Vivo Imaging System and Living Image Software (Caliper Life Sciences, Hopkinton, Massachusetts, USA) was used for image processing. Bioluminescence was measured $15 \mathrm{~min}$ following intraperitoneal injection of $200 \mu \mathrm{L}(150 \mathrm{mg}$ / $\mathrm{kg}$ ) of luciferin. All in vivo experiments were performed twice.

\section{Immunocytokine}

Hu14.18-IL2 IC was provided by Apeiron Biologics and has been previously described. ${ }^{21}$ IT injections of $50 \mu \mathrm{g}$ Hu14.18-IL2 IC in $100 \mu \mathrm{L}$ PBS were delivered daily on days $1-5$.

\section{Resection of primary tumors}

Primary B78 flank tumors were surgically resected on day 8 (where day 1 is the day immunotherapy is initiated) unless otherwise indicated. The surgical resection was performed using sterile technique under isoflurane anesthesia. An incision was made around the tumor mass and the tumor capsule was dissected from underlying tissues. The tumor capsule and overlying skin were removed en bloc and the incision was closed with surgical staples. The surrounding tissues were anesthetized with $0.5 \%$ lidocaine at the conclusion of the procedure and mice were monitored for postoperative pain.

\section{Radiotherapy}

RT was delivered to primary B78 tumors using an Xstrahl Small Animal Radiation Research Platform. Mice were 
immobilized using custom lead jigs that exposed the dorsal right flank as previously described. ${ }^{11}$ Radiation was delivered in one fraction to a maximum dose of $12 \mathrm{~Gy}$.

\section{Flow cytometry}

Resected B78 tumors were mechanically dissociated for 45 min using a gentleMACS dissociator (Miltenyi Biotec) in HBSS supplemented with $1 \mathrm{mg} / \mathrm{mL}$ collagenase type D and $100 \mu \mathrm{g} / \mathrm{mL}$ DNAse I (Sigma-Aldrich) to obtain single cell suspensions. ${ }^{22}$ Ghost Dye Red 780 (Tonbo Biosciences) was used for viability staining. For cell surface staining, cells were preincubated with mouse FC Block anti-mouse CD16/32 (clone 2.4G2, BD Biosciences). After blocking, the cells were labeled with CD3-PE-Cy5 (clone 145-2 C11, Biolegend), CD4-PE-Dazzle594 (clone GK1.5, Biolegend), CD8a-APC-R700 (clone 53-6.7, Biolegend), CD25-BB515 (clone PC61, BD Biosciences), CD45-BV510 (clone 30-F11, Biolegend), GD2-APC (clone 14G2a, Biolegend), and NK1.1-BV421 (clone PK136, Biolegend). Cells were then fixed and permeabilized overnight using Foxp3/Transcription Factor Staining Buffer Set (eBioscience). FoxP3 intracellular staining was then performed prior to flow cytometry. Flow cytometry data were acquired using an Attune NxT Flow Cytometer and analyzed using FlowJo V.10.7.1. The flow cytometry gating strategy is shown in online supplemental figure 1 .

\section{CD4/CD8 Depletion}

Depletion of CD4 T-cells was performed with intraperitoneal (IP) injection of $200 \mu \mathrm{g}$ anti-CD4 mAb (clone GK1.5, BioXCell) in 0.5 mL PBS. Depletion of CD8 T-cells was performed with IP injection of $200 \mu \mathrm{g}$ anti-CD8 mAb (clone 2.43, BioXCell) in $0.5 \mathrm{~mL}$ PBS. Depleting antibodies were administered 2 days prior to intravenous injection of B16 to induce experimental lung metastasis and were continued every 5 days until mice died or reached the end of the experimental period. Confirmation of depletion efficiency was performed via whole blood flow cytometry on the day of B16 injection (online supplemental figure 2).

\section{Histology}

Immunohistochemistry was performed on resected B78 tumors following treatment with neoadjuvant IT-IC or vehicle. Tumors from five mice in each treatment condition were collected. Fresh tumor samples were fixed in $4 \%$ formaldehyde for 24 hours and paraffin embedded. Paraffin embedded tumors were sectioned and antigen retrieval was performed in Tris-EDTA buffer $(10 \mathrm{mM}$ Tris base, $1 \mathrm{mM}$ EDTA, $0.05 \%$ tween 20) at pH 9.0 in a Biocare decloaker (Biocare Medical, Concord, California, USA). Slides were immunostained with primary antibody at $4^{\circ} \mathrm{C}$ overnight using a 1:800 dilution of anti-CD8a (clone D4W2Z, Cell Signaling) or a 1:200 dilution of anti-FoxP3 (clone FJK-16S, Invitrogen). Secondary immunostaining was performed using a dilution of 1:1000 anti-rabbit Alexafluor 488 (Invitrogen) for CD8 and 1:1000 anti-rat Alexafluor 564 (Invitrogen) for FoxP3. Slides were also stained with DAPI
(Invitrogen) to stain nuclei of cells. Representative images were captured of each tumor specimen at $200 \times$ magnification using a LSM710 confocal microscope system with Zen black imaging software (Carl Zeiss). The number of CD8 or FoxP3 positive cells was counted by a blinded observer using representative images from each tumor.

\section{Statistical analysis}

Tumor response was monitored after neoadjuvant therapy and figures show the means and SEs of the tumor volume. Results from each mouse were summarized by the timeweighted average (area under the volume-time curve, calculated using trapezoidal method). Time-weighted averages were compared between treatment groups overall by Kruskal-Wallis tests. If significance was found using the Kruskal-Wallis test, then pairwise comparisons were conducted using Mann-Whitney tests. No p value corrections were applied to the pairwise tests. Complete responders were defined as mice that had zero-volume tumors on the last day of the study and were alive. If significance was found using the $\chi^{2}$ test, then pairwise comparisons were conducted using proportion tests. Survival curves were generated using the Kaplan-Meier method and compared using log-rank tests. Cell quantification from flow cytometry and immunohistochemistry was compared using KruskalWallis tests. $P$ values less than 0.05 were considered statistically significant and are indicated in figures as $* * * p<0.001$; $* * \mathrm{p}<0.01 ; * \mathrm{p}<0.05$; NS, non-significant $(\mathrm{p}>0.05)$. All analyses were performed using GraphPad Prism (GraphPad) or R V.4.0.5 (R Foundation for Statistical Computing).

\section{RESULTS}

\section{Induction of immune memory by neoadjuvant IT-IC}

We have previously shown that IT-IC monotherapy is not sufficient to cure the majority of mice bearing $200 \mathrm{~mm}^{3} \mathrm{~B} 78$ tumors without the addition of RT. ${ }^{11}$ In that prior study, we showed that RT +IT IC could cure $71 \%$ of $200 \mathrm{~mm}^{3} \mathrm{~B} 78$ tumors, while IT-IC alone was far less effective, slowing the growth of the B78 tumors compared with untreated controls, but curing no mice. ${ }^{11}$ For this study, we wished to simulate the clinical neoadjuvant setting, where the clinically evident tumor is resected with margins that would minimize local recurrence. To accomplish this in this B78 model system, we performed pilot studies that showed we could resect tumors from mice bearing $100 \mathrm{~mm}^{3}$ tumors, and without any other therapy these mice would not have local recurrence. In contrast, with tumors of $\sim 200 \mathrm{~mm}^{3}$, some mice not receiving any other treatment than resection were having local recurrence (data not shown). We thus chose to focus on B78 tumors that were $100 \mathrm{~mm}^{3}$. We first confirmed with these $100 \mathrm{~mm}^{3} \mathrm{~B} 78$ tumors, what we had previously published with the $200 \mathrm{~mm}^{3} \mathrm{~B} 78$ tumors; namely that we did not observe a significant benefit in slowing tumor growth or prolonging survival with IT-IC monotherapy whereas RT +IT IC was effective (figure 1A). To determine whether neoadjuvant IT-IC and surgical resection results in immune memory in mice bearing 

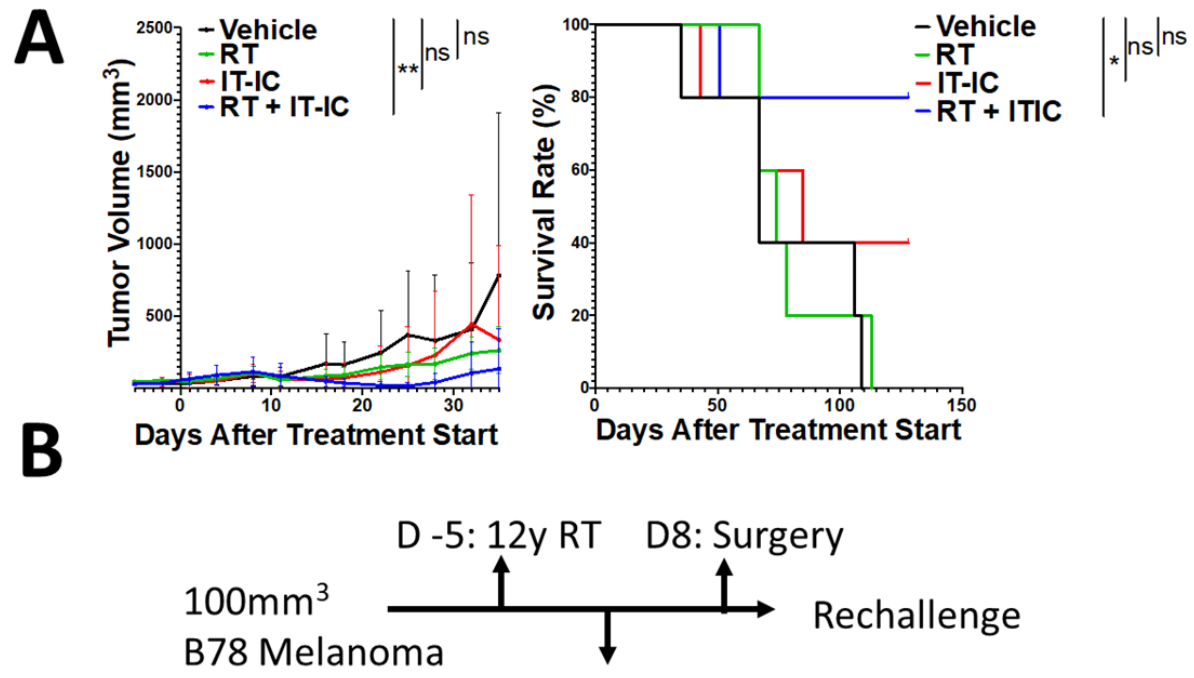

D1-5: IT-IC
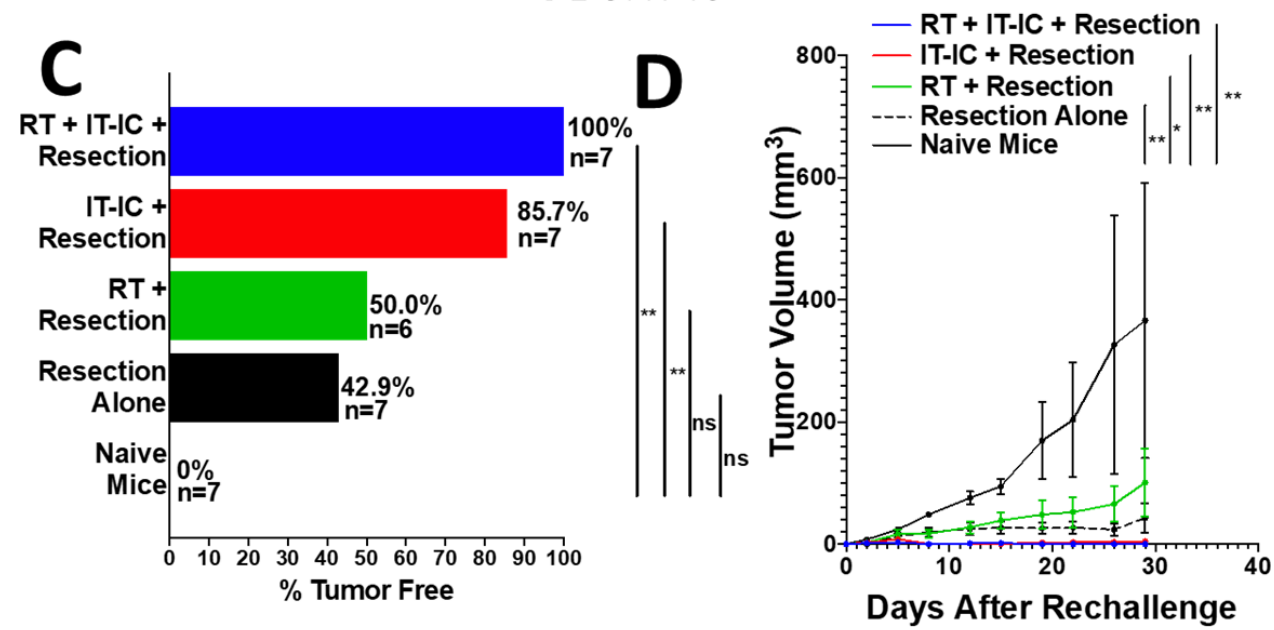

Figure 1 Neoadjuvant IT-IC establishes immunologic memory in B78 melanoma. Intradermal B78 melanoma tumors (mean starting volume $100 \mathrm{~mm}^{3}$ ) were treated with vehicle, RT, IT-IC, or RT +IT IC. (A) Tumor growth and survival of primary tumors that received vehicle, RT, IT-IC, or RT +IT IC, and were not resected surgically, in order to demonstrate the antitumor effects of these four separate therapies on $100 \mathrm{~mm}^{3}$ B78 tumors ( $n=5$ mice per group). (B) Treatment scheme of neoadjuvant immunotherapy approach, that includes resection of all tumors. All mice receiving this regimen, that includes surgical resection, remained tumorfree following resection (were cured). (C-D) Mice cured of their primary tumor by resection alone, or resection with or without RT and/or IT-IC were rechallenged in the contralateral flank with B78 melanoma at day 30 following the start of neoadjuvant immunotherapy, along with simultaneous implantation of B78 into separate naïve mice; tumor growth over 30 days and \% tumor-free mice at day 30 , are shown ( $n=7$ mice per group). All experiments performed in duplicate and a representative single experiment is shown. NS, non-significant; ${ }^{\star} \mathrm{p}<0.05$; ${ }^{* \star} \mathrm{p}<0.01$; ${ }^{\star \star \star} \mathrm{p}<0.001$. IT-IC, intratumoral immunocytokine; RT, radiotherapy; y, years.

immunologically cold B78 melanoma tumors, we used the treatment regimen summarized in figure $1 \mathrm{~B}$.

Despite its limited efficacy against $\sim 100 \mathrm{~mm}^{3}$ B 78 flank tumors, we hypothesized that IT-IC might still generate an effective systemic immune response when used as an in-situ tumor vaccine prior to complete surgical resection of the tumor. IT-IC, with or without RT, was administered in a similar fashion to $100 \mathrm{~mm}^{3} \mathrm{~B} 78$ tumors. Mice then underwent surgical resection of the primary flank tumor 3 days following the final dose of IT-IC. Following neoadjuvant therapy and surgical resection, mice were rechallenged on the contralateral flank with B78 tumor cells. We observed that mice cured with neoadjuvant IT-IC and surgery had slower rechallenge tumor growth and a significantly higher rate of rechallenge rejection than naive mice, almost as high as mice cured with $\mathrm{RT}+\mathrm{IT}$ IC (figure 1C,D). Individual tumor growth curves for the data shown in figure $1 \mathrm{D}$ are shown in online supplemental figure 2A.

The flank tumors of mice treated with neoadjuvant immunotherapy were analyzed by flow cytometry (figure 2A). We found that neoadjuvant IT-IC was associated with significantly increased infiltration of CD8 T-cells and a higher CD8:regulatory $\mathrm{T}$ (Treg) cells ratio compared with mice that received vehicle alone, though these differences were less pronounced compared with mice that received combination RT and IT-IC. The significant differences in CD8 T-cells and Tregs were 

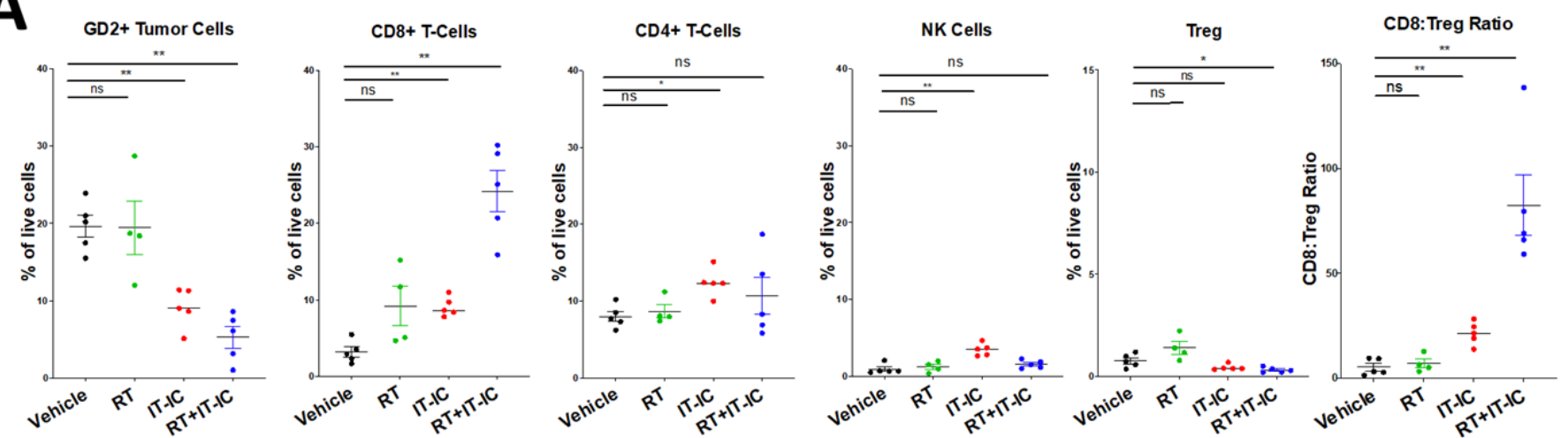

B

\section{CD8 ${ }^{+} \mathrm{T}$ cells}
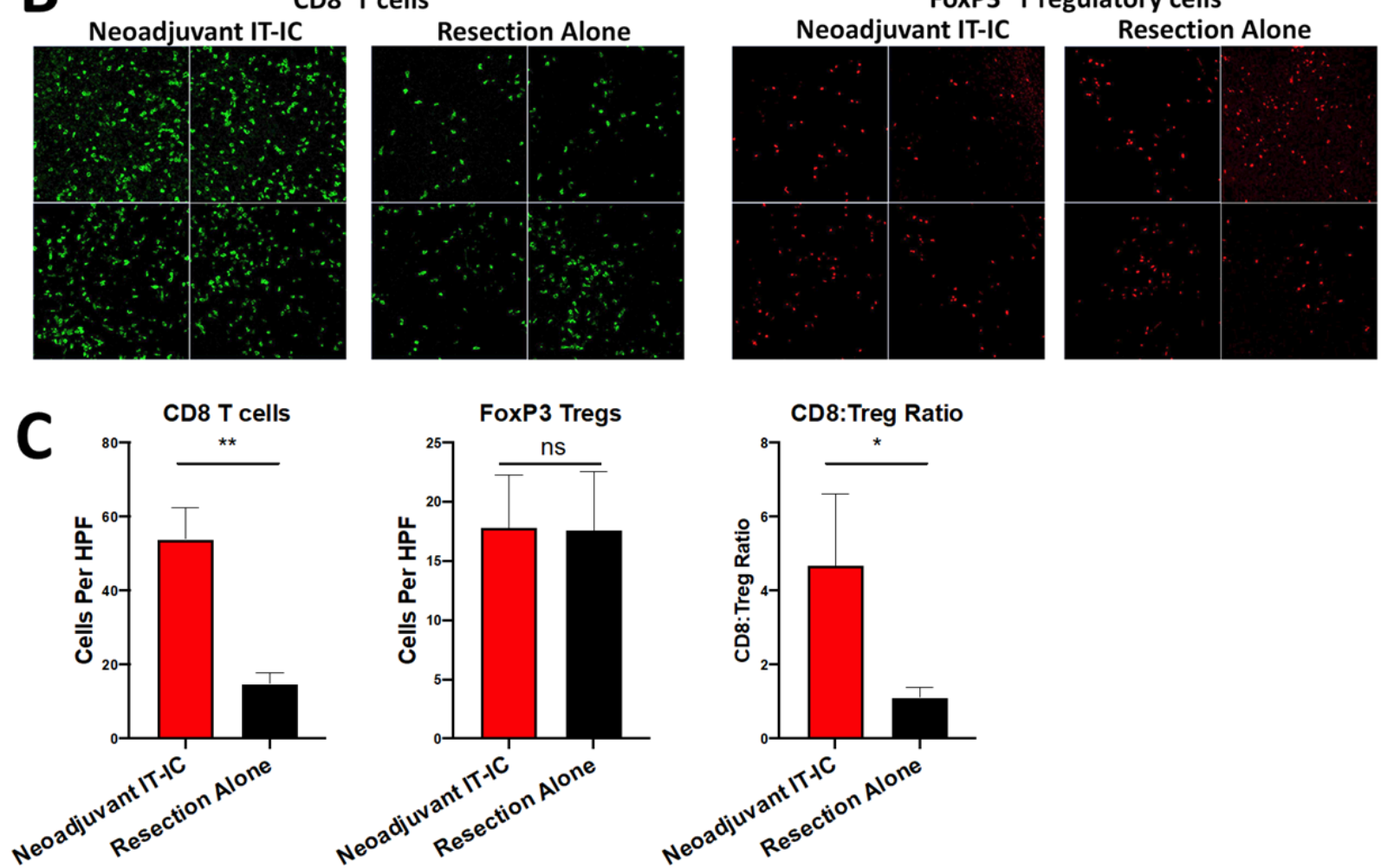

Figure 2 Neoadjuvant IT-IC is associated with increased infiltration of CD8 T cells and a higher CD8:Treg ratio. (A) The immune infiltrate of untreated tumors or tumors treated with neoadjuvant RT, IT-IC, or RT +IT IC as assessed by flow cytometry of tumor digests ( $n=5$ mice per group). (B) Immunohistochemistry for CD8-positive T-cells (in green, left two panels) and FoxP3-positive T regulatory cells (in red, right two panels). (C) Cells per high power field as counted from the immunohistochemistry experiments shown in B. Flow cytometry experiments were performed in duplicate, and a representative single experiment is shown. Histology was performed on tumor specimens from five mice per treatment condition, and representative blinded specimens were obtained for the photos, and for blinded enumeration. All experiments performed in duplicate and a representative single experiment is shown. NS, non-significant; ${ }^{\star} \mathrm{p}<0.05 ;{ }^{* \star} \mathrm{p}<0.01 ;{ }^{* \star \star} \mathrm{p}<0.001$. HPF, high power field; IT-IC, intratumoral immunocytokine; NK, natural killer; RT, radiotherapy; Treg, regulatory T cells.

confirmed via immunohistochemistry of treated tumors (figure 2B,C).

\section{Timing of rechallenge and resection following neoadjuvant IT-IC}

Our next step was to assess the impact of timing of surgery and rechallenge on the development of memory induced by neoadjuvant IT-IC. We assessed the impact of surgical timing on the development of memory using the regimen indicated in figure $3 \mathrm{~A}$. Mice were administered neoadjuvant IT-IC and underwent surgical resection at either Day 8 or Day 15. The resected tumors were analyzed by flow cytometry. We found significantly increased infiltrating CD8 T cells at Day 8, but no longer at Day 15 (figure 3B). The infiltrating Treg cells were significantly reduced at Day 15 and the CD8:Treg ratio was significantly elevated compared with mice that received vehicle at both Day 8 and Day 15. Equivalent immunologic memory and rechallenge tumor growth was observed with early and late surgery following neoadjuvant IT-IC (figure 3C,D). 


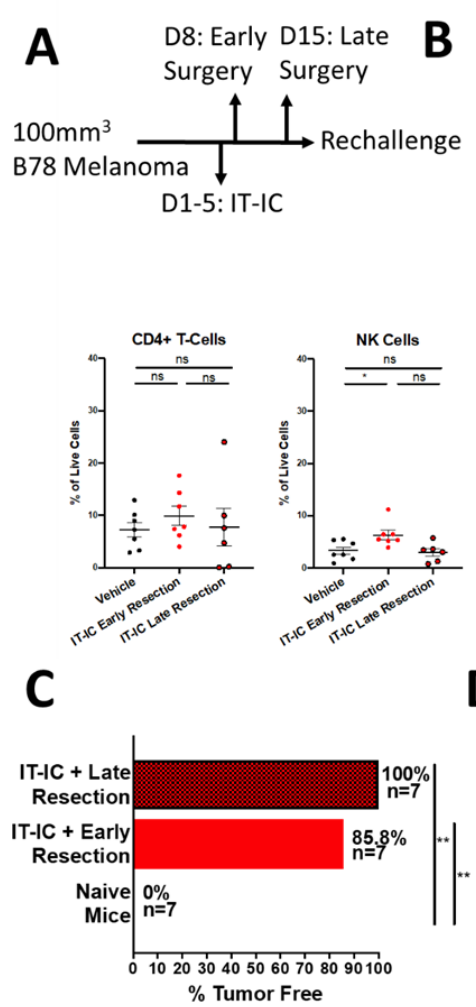

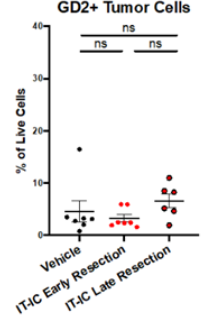
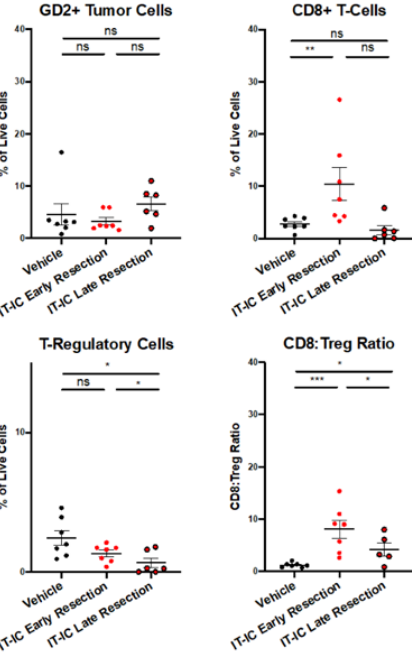

CD8: Treg Ratio
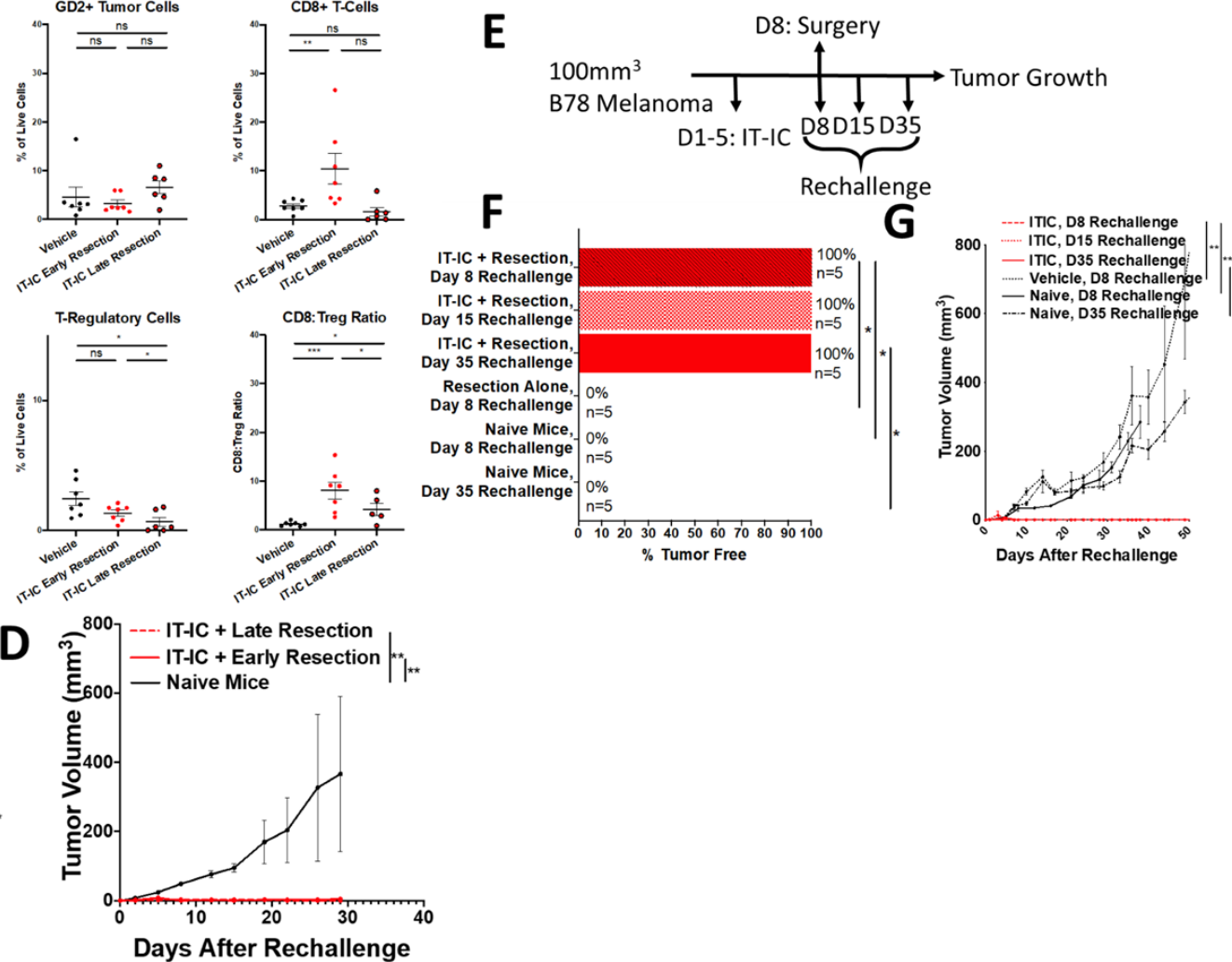

Figure 3 Impact of surgical timing and rechallenge timing on the development of immunologic memory. (A) Early and late surgical resection following neoadjuvant IT-IC was performed according to the depicted scheme, with IT-IC given on day 1-5 and resection done either on day 8 or day 15. (B) The immune infiltrate of tumors resected following early and late surgery as assessed by flow cytometry on tumor digests ( $n=5$ mice per group). (C,D) Mice cured of their primary tumor by early or late resection following IT-IC were rechallenged in the contralateral flank with B78 melanoma at day 30 following the start of neoadjuvant IT-IC, along with simultaneous implantation of B78 into naïve mice; \% tumor-free mice at day 30 and tumor growth are shown ( $n=7$ mice per group). (Note, in online supplemental figure 3 the separate lines for the data with early or late resection following IT-IC are superimposed, and thus appear as a single line). (E) The timing of the systemic response was assessed by rechallenge of B78 on the contralateral flank on day 8 , day 15 or day 35 , according to the scheme depicted. (F,G). Mice cured of their primary tumor by early resection following IT-IC were rechallenged in the contralateral flank with B78 melanoma at day 8 , day 15 or day 35 following the start of neoadjuvant IT-IC, along with simultaneous implantation of B78 on day 8 or day 35 into naïve mice, and on day 8 into mice cured by resection alone; tumor growth over 30 days and \% tumor-free mice at day 30 , are shown ( $n=5$ mice per group). All experiments performed in duplicate and a representative single experiment is shown. NS, nonsignificant; ${ }^{*} \mathrm{p}<0.05 ;{ }^{* *} \mathrm{p}<0.01 ;{ }^{* *} \mathrm{p}<0.001$. IT-IC, intratumoral immunocytokine; NK, natural killer.

To determine whether an effective systemic immune response was present as early as the time of surgery, we used the treatment and rechallenge regimen indicated in figure 3E. Following neoadjuvant IT-IC and surgical resection, mice were rechallenged with contralateral B78 flank tumors on Day 8, Day 15, and Day 35. We found that all mice treated with neoadjuvant IT-IC rejected contralateral rechallenge on Day 8, while all naive mice and mice that received surgery alone had tumor engraftment (figure 3F). Mice treated with neoadjuvant IT-IC also rejected rechallenge at Day 15 and Day 35. Rechallenge tumor growth is shown in figure $3 \mathrm{G}$ and the individual tumor curves following rechallenge are shown in online supplemental figure $2 \mathrm{~B}$.

\section{Development of memory against lung metastasis following neoadjuvant IT-IC}

We sought to determine whether the immune memory generated by neoadjuvant IT-IC was sufficient to reject distant recurrence using our previously described B16 lung metastasis model. ${ }^{11}$ B16 melanoma lacks endogenous expression of GD2, thus B16-GD2 cells were generated to express GD2. Before proceeding with this GD2-independent metastasis model, we first demonstrated that mice previously cured via neoadjuvant IT-IC and surgery reject intravenously injected B16-GD2 cells (online supplemental figure 3A-C).

We have previously shown that mice bearing B78 tumors rendered disease-free following RT + IT-IC have developed tumor-specific $\mathrm{T}$ cell memory and antibody responses against the non-GD2-expressing B16 melanoma, indicating that antigens other than GD2 are recognized by the $\mathrm{T}$ and $\mathrm{B}$ cells of these immune mice. ${ }^{11} 23$ Thus, we next tested whether treated mice would reject intravenous rechallenge with B16 melanoma according to the treatment scheme depicted in figure 4A. Because we had observed local wound toxicity from the combination 
A

B

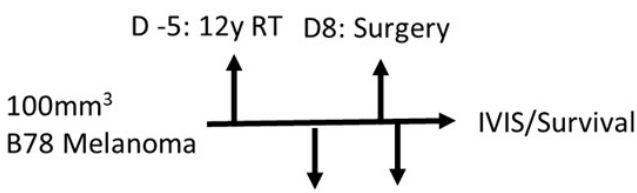

D1-5: IT-IC D9: B16 Tail Vein Injection
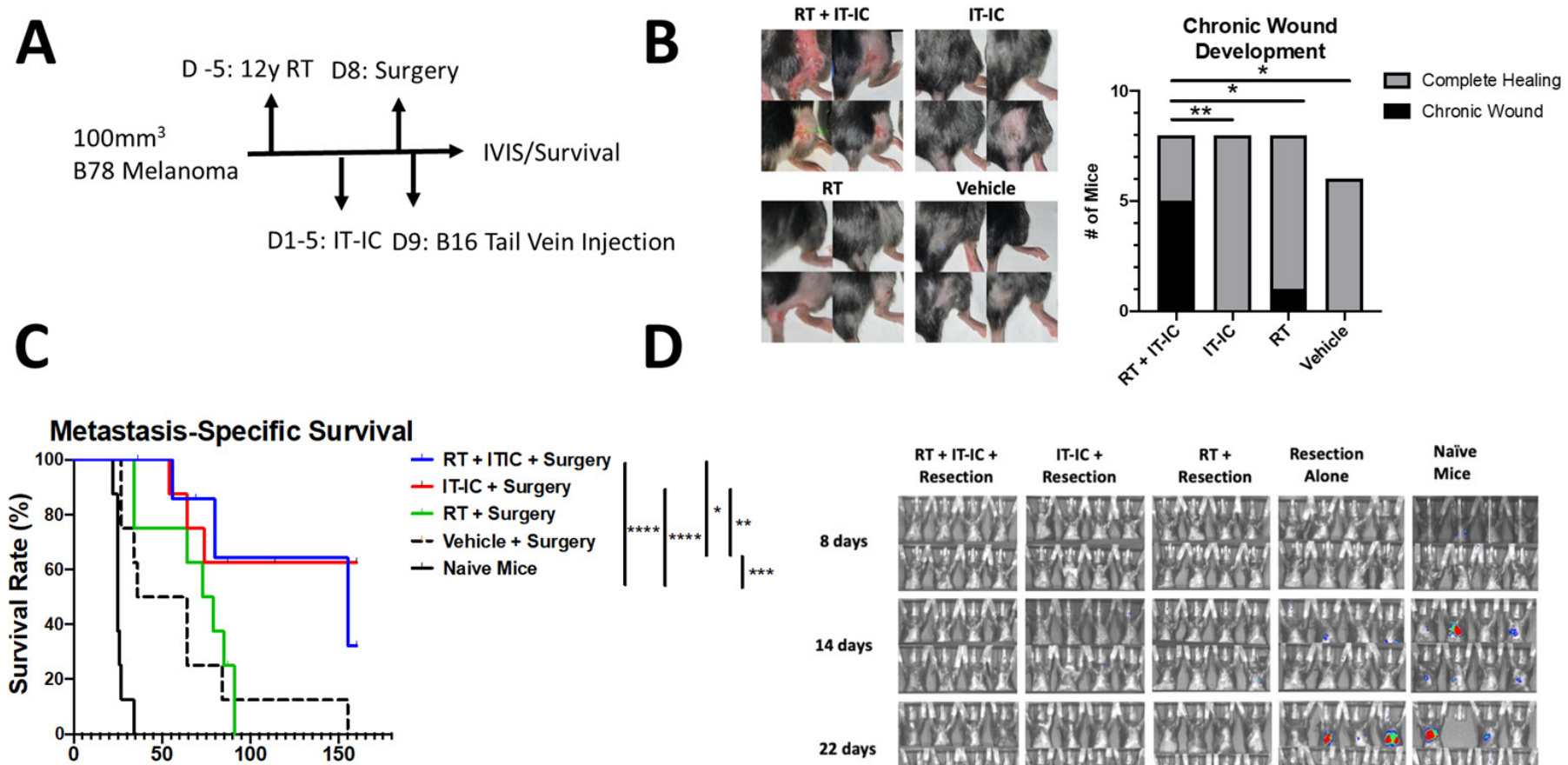

Days After IV Injection of B16 cells
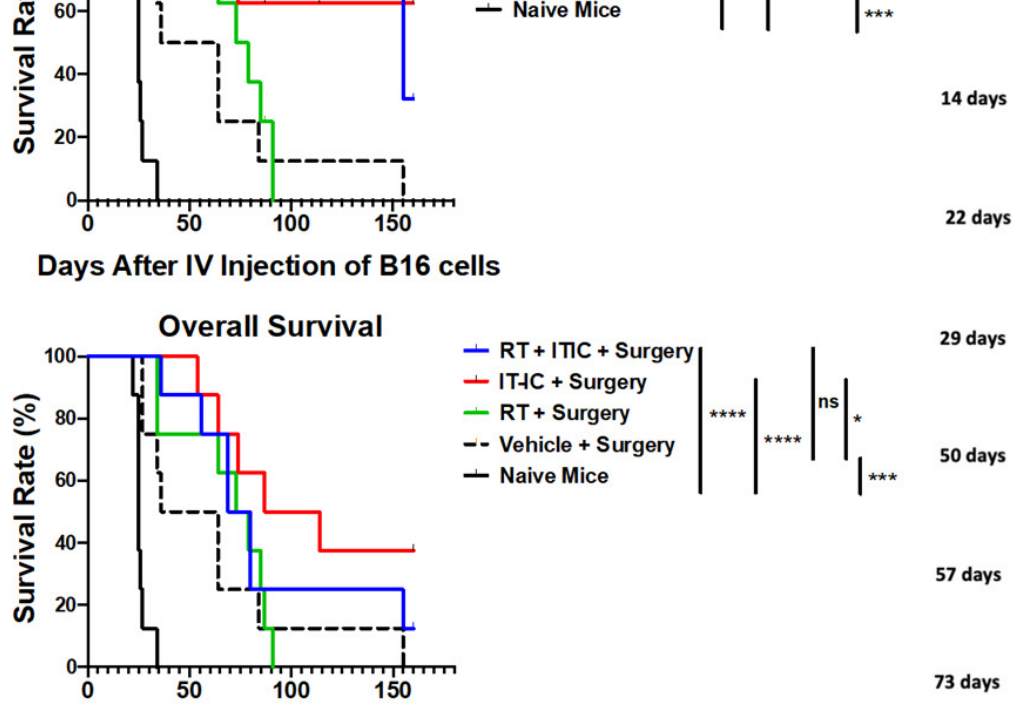

22 days
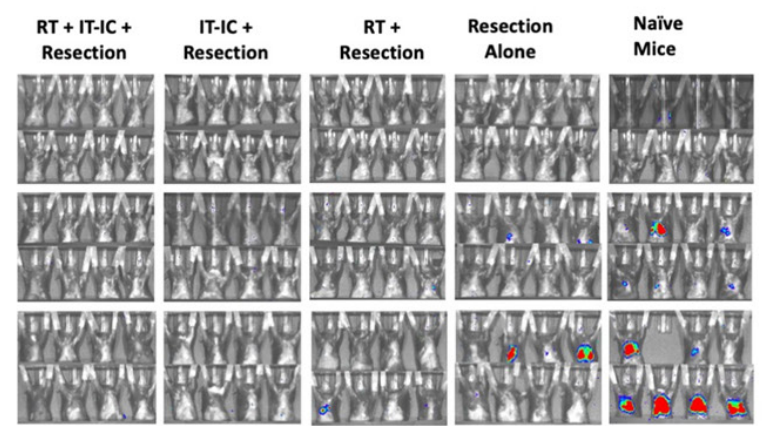

9 days
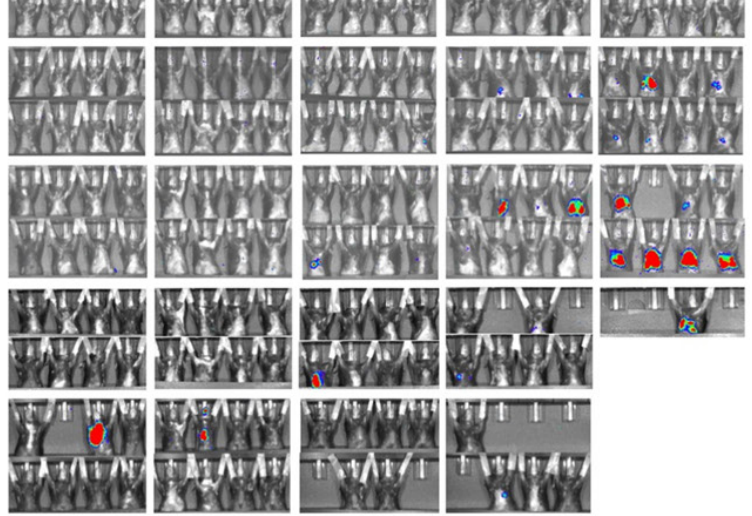

ays After IV Injection of B16 cells

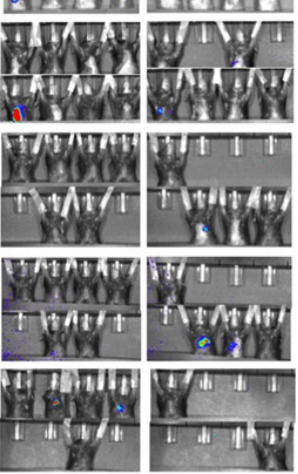

Figure 4 Neoadjuvant IT-IC inhibits establishment of B16 lung metastasis. (A) Mice bearing B78 melanoma tumors were untreated or treated with neoadjuvant RT, IT-IC, or RT + IT IC and tumors were subsequently resected. Mice were challenged IV with B16 cells on the day following surgery to induce experimental lung metastasis. (B) Assessment of chronic wound formation following surgical resection, performed at day 40 following the start of neoadjuvant immunotherapy; representative images from each group are shown, and the bar graph clarifies the number of mice in each group that had complete healing of their resection wound or had a chronic non-healed wound. (C) Metastasis-specific and overall survival following challenge with IV B16 induced experimental lung metastasis ( $n=8$ mice per group). (D) Representative IVIS images of mice from each group following challenge with IV B16 experimental lung metastasis. All experiments performed in duplicate and a representative single experiment is shown. NS, non-significant; ${ }^{*} \mathrm{p}<0.05$; ${ }^{\star \star} \mathrm{p}<0.01$; ${ }^{\star \star \star} \mathrm{p}<0.001$. IT-IC, intratumoral immunocytokine; IV, intravenous; RT, radiotherapy; y, years.

of radiation and neoadjuvant IT-IC, we quantified the frequency of local wound complications present 40 days following the start of therapy. We found that RT, neoadjuvant IT-IC, and surgery resulted in chronic surgical wounds in most treated mice (figure 4B). No chronic wounds were observed in mice that received neoadjuvant IT-IC and surgery alone.

Survival curves and IVIS images following B16 tail vein injection are shown in figure 4C,D. Metastasis-specific and overall survival were both recorded due to several mice in the radiation plus neoadjuvant IT-IC group requiring euthanasia due to local wound complications. We observed improved survival in mice treated with neoadjuvant IT-IC compared with naïve mice or mice that received surgery alone.

\section{Mediators of B16 lung metastasis rejection}

We have previously shown that immune mediated rejection of B78 rechallenge is T-cell dependent. ${ }^{11}$ We sought to characterize the mechanism of B16 lung metastasis rejection following neoadjuvant IT-IC. We performed CD4 and CD8 T-cell depletion according to the scheme depicted in figure 5A. Successful depletion was confirmed by flow cytometry (online supplemental figure 4A,B). CD4 and combined CD4/8 depletion was found to be associated with shorter survival compared with non-depleted, 


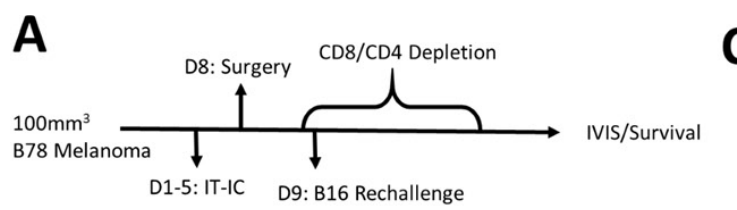

B

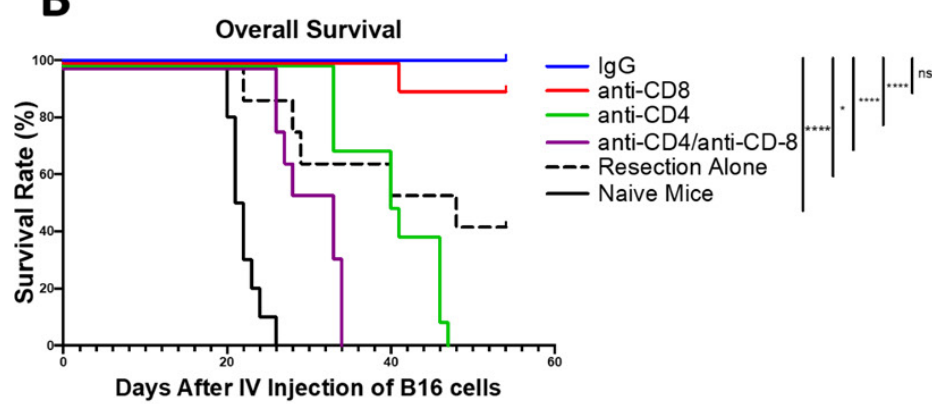

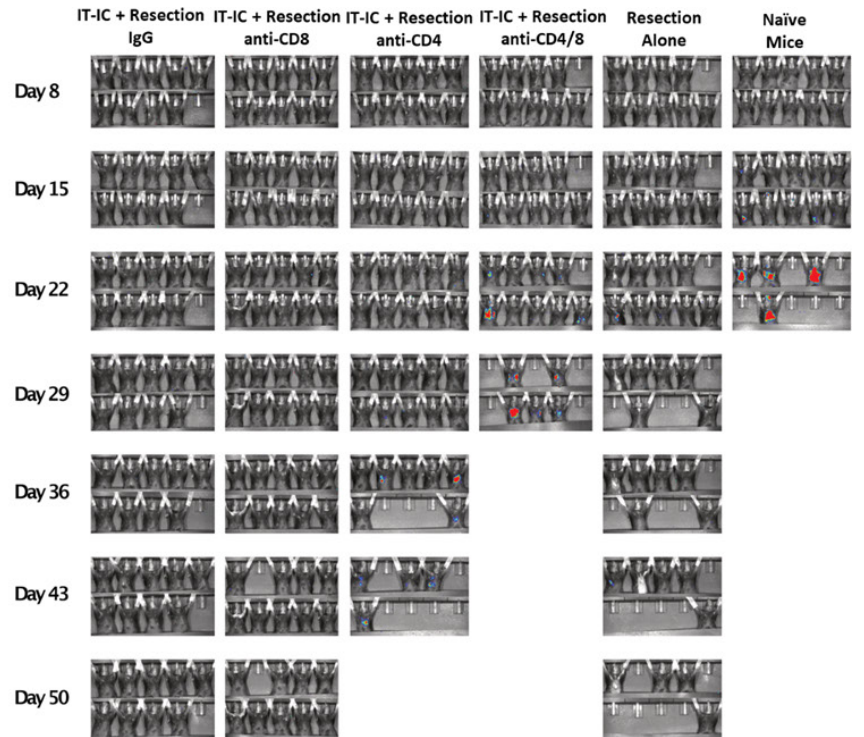

Figure 5 Rejection of B16 rechallenge following neoadjuvant IT-IC is dependent on CD4 T cells. (A) Mice bearing B16 melanoma tumors were treated with neoadjuvant IT-IC and day 8 resection; they subsequently underwent CD8/4 T cell depletion beginning 2 days prior to rechallenge with IV injection of B16 to induce experimental lung metastasis. Depletion was continued every 5 days until mice died or reached the end of the study period. (B) Overall survival following IV injection of B16 cells ( $n=10$ mice per group). (C) Representative IVIS images of mice following challenge with B16 lung metastasis. All experiments performed in duplicate and a representative single experiment is shown. NS, non-significant; ${ }^{*} \mathrm{p}<0.05 ;{ }^{* *} \mathrm{p}<0.01$; ${ }^{* \star *} \mathrm{p}<0.001$. IT-IC, intratumoral immunocytokine; IV, intravenous

IT-IC treated mice (figure 5B,C), implicating CD4 T cells as being involved in this response. This is consistent with prior work using a separate immunotherapy in B78 melanoma. ${ }^{24}$ There was no significant difference in survival with $\mathrm{CD} 8$ depletion compared with non-depleted mice.

\section{DISCUSSION}

We demonstrate that a GD2-based neoadjuvant tumor vaccination strategy using an IL2-linked IT-IC can generate systemic immunity in a murine model of melanoma. This effect is present as early as the day of surgery and is capable of inhibiting engraftment of non-GD2 expressing melanoma lung metastasis. These results are in agreement with our previous studies in the B78 model showing antigen-specific memory in mice cured with RT and IT-IC and rechallenged with subcutaneous tumors, in that cured mice rejected GD2-deficient B16 melanoma cells (parental to B78) but not unrelated syngeneic Panc02 pancreatic tumor cells. ${ }^{11}$ Importantly, this new strategy also avoids the local surgical wound toxicity of combined RT and IT-IC. Overall, our results demonstrate a potential novel application of an in situ tumor vaccination approach currently under clinical investigation in melanoma (NCT03958383).

Previous studies provide significant guidance regarding the optimal design of a neoadjuvant immunotherapy regimen. Liu et al demonstrated in $4 \mathrm{~T} 1.2$ breast cancer that a short duration (4-5 days) between initiation of immunotherapy and surgical resection was optimal in a neoadjuvant immunotherapy regimen that included checkpoint inhibition. ${ }^{8}$ However, efficacy was decreased if the therapy was administered up until the time of surgery. The peak expansion of peripheral tumor-specific CD8 T-cells occurred shortly after the completion of neoadjuvant therapy. Our regimen is consistent with the optimal timing described in this Liu et al study; we observed the highest infiltration of CD8 T-cells occurred immediately following the completion of immunotherapy. The timing used for our regimen is also similar to a previous study of adenovirus-based neoadjuvant tumor vaccination in B16 melanoma, where vaccination was effective when given in the days immediately preceding surgery but not in the adjuvant setting. ${ }^{10}$

We can consider when a neoadjuvant tumor vaccination strategy might be useful clinically. In patients with thin melanoma on initial biopsy and no adverse features, the risk of nodal or distant disease is low $(<5 \%)$, and wide local excision is sufficient treatment. ${ }^{25}{ }^{26}$ The risk of nodal metastasis increases with tumor depth at diagnosis; approximately $40 \%$ of patients with thick melanoma ( $>4$ $\mathrm{mm}$ ) have nodal disease. ${ }^{27}$ Even in the era of adjuvant checkpoint inhibition, the survival of patients with a positive sentinel lymph node is reduced due to eventual distant recurrence. ${ }^{28} \mathrm{~A}$ short-course neoadjuvant tumor vaccination strategy given before surgery in patients with high-risk intermediate or thick melanoma on initial biopsy might generate a systemic response against distant micrometastasis, potentially preventing progression or further dissemination. The pathologic response might also provide important prognostic information, as has been observed in a phase I trial of this immunocytokine when delivered intravenously (IV-IC) ${ }^{29}$ 
We observed that a combination of neoadjuvant RT and IT-IC is similarly effective in generating a systemic immune response to RT and IT-IC without the post-therapy tumor resection, and is more effective against the primary tumor than is IT-IC without RT, the latter of which has been demonstrated previously. ${ }^{11}$ However, IT-IC monotherapy might be more attractive as a neoadjuvant tumor vaccination approach. Despite the higher risk of nodal positivity in high-risk intermediate or thick melanoma, the majority of patients have a favorable outcome. In these patients, local wound complications similar to those we observed with RT and IT-IC may outweigh the potential therapeutic benefit of combination therapy. The reduced local toxicity of IT-IC monotherapy might allow it to be safely applied in this broad patient population. While the systemic toxicity of IT-IC is currently being assessed in a phase I trial of patients with stage IV melanoma (NCT03958383), the toxicity of IV-IC was reversible and tolerable in previous clinical trials. ${ }^{29} 30$

Future preclinical studies can investigate the prognostic value of neoadjuvant IT-IC. While we observed increased infiltration of CD8 Tcells in the tumor microenvironment following tumor resection, CD8 T-cells were not required for the memory responses against B16 lung metastases. However, an association between CD8 T-cell infiltration into tumor and improved outcome was observed in a phase I clinical trial of IV-IC when given before surgery for melanoma; further analysis of these resection specimens revealed that several immune signatures were associated with outcome. ${ }^{29} 31$

While the B16 melanoma expresses low levels of MHC-I, the B78 melanoma that is derived from B16 has virtually no MHC-I expression. ${ }^{24}{ }^{32}$ We have previously demonstrated that the response of $\mathrm{B} 78$ melanoma to this $\mathrm{RT}+\mathrm{IT}$ IC regimen is $\mathrm{T}$ cell dependent, for both the primary response and for the rejection of rechallenged tumors by mice cured using this regimen. ${ }^{11}$ In this present report we show that CD4 T cells play a more prominent role than CD8 $\mathrm{T}$ cells in preventing regrowth of B16 lung metastases after rechallenge. The relative roles of MHC-I and MHCII, and of CD4 and CD8 T cells, in these responses against the primary tumors and in the setting of rechallenge will be presented in a separate manuscript (Erbe AK et al, In Preparation). Additional analysis of tumor treated with neoadjuvant IT-IC, including single-cell RNA sequencing, might reveal predictors of response in this model. Such information may eventually prove useful for prognostic purposes and for identifying those high-risk patients who might benefit from further adjuvant treatment.

\section{Conclusion}

We show here that short-course neoadjuvant IT-IC generates immunologic memory capable of preventing distant metastasis despite limited efficacy against large primary melanoma tumors. By combining neoadjuvant tumor vaccination and surgery, the toxicity of local RT was avoided. Low-toxicity local immunotherapy is particularly attractive in this setting, where most patients do not go on to have distant metastasis even in the absence of immunotherapy. These preclinical data support further investigation regarding the use of neoadjuvant IT-IC in patients with melanoma at high risk for occult distant disease.

Contributors TJA performed experiments, analyzed and interpreted data, and was the primary author of this manuscript. DK, MR, and AS assisted in animal studies, including drug administration and tumor measurements. AF and AE assisted in flow cytometry. VS assisted with histologic analysis and immunohistochemistry. JB assisted with statistical analyses. SDG originally created the immunocytokine and the antibody studied. TJA, AE, ALR, and PMS were key contributors to the experimental concept and design. PMS was the guarantor of the study. All authors contributed to the writing of the manuscript and approved the final manuscript.

Funding This research was supported by Midwest Athletes Against Childhood Cancer; Stand Up To Cancer (SU2C); the St. Baldrick's Foundation; American Association of Cancer Research; University of Wisconsin Carbone Cancer Center; The Crawdaddy Foundation and Children's Neuroblastoma Cancer Foundation. Stand Up To Cancer is a program of the Entertainment Industry Foundation. Research grants are administered by the American Association for Cancer Research, the scientific partner of SU2C. This research was also supported in part by public health service grants U54 CA232568, CA014520, CA166105, CA197078, CA233102, CA250972 and CA090217 from the National Cancer Institute; the National Institutes of Health and the Department of Health and Human Services. Its content is solely the responsibility of the authors and does not necessarily represent the official views of the National Cancer Institute.

Competing interests VS declares employment in Arrowhead Pharmaceuticals. SDG declares employment and ownership interests in Provenance Biopharmaceuticals.

\section{Patient consent for publication Not applicable.}

Ethics approval Mice were used in accordance with the Guide for Care and Use of Laboratory Animals.

Provenance and peer review Not commissioned; externally peer reviewed.

Data availability statement Data are available upon reasonable request. The data sets generated and/or analyzed during the current study are not publicly available due to their relevance only for the experiments presented here but are available from the corresponding author on reasonable request.

Supplemental material This content has been supplied by the author(s). It has not been vetted by BMJ Publishing Group Limited (BMJ) and may not have been peer-reviewed. Any opinions or recommendations discussed are solely those of the author(s) and are not endorsed by BMJ. BMJ disclaims all liability and responsibility arising from any reliance placed on the content. Where the content includes any translated material, BMJ does not warrant the accuracy and reliability of the translations (including but not limited to local regulations, clinical guidelines, terminology, drug names and drug dosages), and is not responsible for any error and/or omissions arising from translation and adaptation or otherwise.

Open access This is an open access article distributed in accordance with the Creative Commons Attribution Non Commercial (CC BY-NC 4.0) license, which permits others to distribute, remix, adapt, build upon this work non-commercially, and license their derivative works on different terms, provided the original work is properly cited, appropriate credit is given, any changes made indicated, and the use is non-commercial. See http://creativecommons.org/licenses/by-nc/4.0/.

\section{ORCID iD}

Paul M Sondel http://orcid.org/0000-0002-0981-8875

\section{REFERENCES}

1 Ando $\mathrm{N}$, Kato $\mathrm{H}$, Igaki $\mathrm{H}$, et al. A randomized trial comparing postoperative adjuvant chemotherapy with cisplatin and 5-fluorouracil versus preoperative chemotherapy for localized advanced squamous cell carcinoma of the thoracic esophagus (JCOG9907). Ann Surg Oncol 2012;19:68-74.

2 Shapiro J, van Lanschot JJB, Hulshof MCCM, et al. Neoadjuvant chemoradiotherapy plus surgery versus surgery alone for oesophageal or junctional cancer (CROSS): long-term results of a randomised controlled trial. Lancet Oncol 2015;16:1090-8.

3 Sauer R, Becker H, Hohenberger W, et al. Preoperative versus postoperative chemoradiotherapy for rectal cancer. N Engl J Med 2004;351:1731-40. 
4 Rosell R, Gomez-Codina J, Camps C, et al. A randomized trial comparing preoperative chemotherapy plus surgery with surgery alone in patients with non-small-cell lung cancer. N Engl J Med Overseas Ed 1994;330:153-8.

5 Fisher B, Bryant J, Wolmark N, et al. Effect of preoperative chemotherapy on the outcome of women with operable breast cancer. J Clin Oncol 1998;16:2672-85.

6 Topalian SL, Taube JM, Pardoll DM. Neoadjuvant checkpoint blockade for cancer immunotherapy. Science 2020;367:aax0182.

7 Liu J, Blake SJ, Yong MCR, et al. Improved efficacy of neoadjuvant compared to adjuvant immunotherapy to eradicate metastatic disease. Cancer Discov 2016;6:1382-99.

8 Liu J, O'Donnell JS, Yan J, et al. Timing of neoadjuvant immunotherapy in relation to surgery is crucial for outcome. Oncoimmunology 2019;8:e1581530.

9 Arora A, Su G, Mathiowitz E, et al. Neoadjuvant intratumoral cytokine-loaded microspheres are superior to postoperative autologous cellular vaccines in generating systemic anti-tumor immunity. J Surg Oncol 2006;94:403-12.

10 Grinshtein N, Bridle B, Wan Y, et al. Neoadjuvant vaccination provides superior protection against tumor relapse following surgery compared with adjuvant vaccination. Cancer Res 2009;69:3979-85.

11 Morris ZS, Guy El, Francis DM, et al. In situ tumor vaccination by combining local radiation and tumor-specific antibody or immunocytokine treatments. Cancer Res 2016;76:3929-41.

12 Morris ZS, Guy El, Werner LR, et al. Tumor-specificinhibition of In Situ vaccination by distant untreated tumor sites. Cancer Immunol Res 2018;6:825-34.

13 Johnson EE, Lum HD, Rakhmilevich AL, et al. Intratumoral immunocytokine treatment results in enhanced antitumor effects. Cancer Immunol Immunother 2008:57:1891-902.

14 Weide B, Eigentler TK, Pflugfelder A, et al. Intralesional treatment of stage III metastatic melanoma patients with L19-IL2 results in sustained clinical and systemic immunologic responses. Cancer Immunol Res 2014;2:668-78.

15 Radny P, Caroli UM, Bauer J, et al. Phase II trial of intralesional therapy with interleukin-2 in soft-tissue melanoma metastases. $\mathrm{Br} \mathrm{J}$ Cancer 2003;89:1620-6.

16 Weide B, Eigentler TK, Pflugfelder A, et al. Survival after intratumoral interleukin-2 treatment of 72 melanoma patients and response upon the first chemotherapy during follow-up. Cancer Immunol Immunother 2011;60:487-93.

17 Rashid OM, Nagahashi M, Ramachandran S, et al. Resection of the primary tumor improves survival in metastatic breast cancer by reducing overall tumor burden. Surgery 2013;153:771-8.

18 Becker JC, Varki N, Gillies SD, et al. An antibody-interleukin 2 fusion protein overcomes tumor heterogeneity by induction of a cellular immune response. Proc Natl Acad Sci U S A 1996;93:7826-31.
19 Haraguchi M, Yamashiro S, Yamamoto A, et al. Isolation of GD3 synthase gene by expression cloning of GM3 alpha-2,8sialyltransferase cDNA using anti-GD2 monoclonal antibody. Proc Natl Acad Sci U S A 1994:91:10455-9.

20 Silagi S. Control of pigment production in mouse melanoma cells in vitro. evocation and maintenance. J Cell Biol 1969;43:263.

21 Gillies SD, Reilly EB, Lo KM, et al. Antibody-targeted interleukin 2 stimulates T-cell killing of autologous tumor cells. Proc Natl Acad Sci U S A 1992;89:1428-32.

22 Rakhmilevich AL, Felder M, Lever L, et al. Effective combination of innate and adaptive immunotherapeutic approaches in a mouse melanoma model. J Immunol 2017;198:1575-84.

23 Baniel CC, Heinze CM, Hoefges A. In situ vaccine plus checkpoint blockade induces memory humoral response. Front Immunol 2020;11:1610.

24 Levitsky HI, Lazenby A, Hayashi RJ, et al. In vivo priming of two distinct antitumor effector populations: the role of $\mathrm{MHC}$ class I expression. J Exp Med 1994;179:1215-24.

25 Sinnamon AJ, Neuwirth MG, Yalamanchi P, et al. Association between patient age and lymph node positivity in thin melanoma. JAMA Dermatol 2017;153:866-73.

26 National Comprehensive Cancer Network. Cutaneous melanoma (version 1.2020). Available: https://www.nccn.org/professionals/ physician_gls/pdf/cutaneous_melanoma.pdf [Accessed 28 Mar 2020].

27 Mozzillo N, Pennacchioli E, Gandini S, et al. Sentinel node biopsy in thin and thick melanoma. Ann Surg Oncol 2013;20:2780-6.

28 Eggermont AMM, Chiarion-Sileni V, Grob J-J, et al. Adjuvant ipilimumab versus placebo after complete resection of stage II melanoma: long-term follow-up results of the European organisation for research and treatment of cancer 18071 double-blind phase 3 randomised trial. Eur J Cancer 2019;119:1-10.

29 Albertini MR, Yang RK, Ranheim EA, et al. Pilot trial of the hu14.18IL2 immunocytokine in patients with completely resectable recurrent stage III or stage IV melanoma. Cancer Immunol Immunother 2018;67:1647-58.

30 King DM, Albertini MR, Schalch $\mathrm{H}$, et al. Phase I clinical trial of the immunocytokine EMD 273063 in melanoma patients. J Clin Oncol 2004;22:4463-73.

31 Yang RK, Kuznetsov IB, Ranheim EA, et al. Outcome-Related signatures identified by whole transcriptome sequencing of resectable stage III/IV melanoma evaluated after starting Hu14.18IL2. Clin Cancer Res 2020;26:3296-306.

32 Lasek W, Mackiewicz A, Czajka A, et al. Antitumor effects of the combination therapy with TNF- $\alpha$ gene-modified tumor cells and interleukin 12 in a melanoma model in mice. Cancer Gene Ther 2000;7:1581-90. 